\title{
Cuando lo mínimo es máximo. Los minimizadores escalares y la polaridad negativa
}

\author{
por Rosabel San Segundo Cachero \\ ORCID: 0000-0003-3867-3422 \\ Universidad de Zaragoza
}

\section{INTRODUCCIÓN}

Tos minimizadores constituyen un repositorio fundamental en las L lenguas para la expresión de cantidades mínimas y, por su valor escalar, tras un proceso de gramaticalización, se convierten en cuantificadores que bajo el alcance de la negación pueden reanalizarse como marcadores negativos de carácter enfático.

Las unidades léxicas que integran el grupo de los minimizadores son muy variadas y su comportamiento está en buena parte condicionado por las restricciones combinatorias que impone su contenido semántico y por el grado de gramaticalización que presentan, aspecto que se refleja en la capacidad para funcionar autónomamente con sintagmas nominales escuetos en contextos negativos, lo que convierte a algunos minimizadores en términos de polaridad negativa fuertes.

\footnotetext{
I Agradezco a Ramón de Andrés su interés en mi trabajo y a los revisores anónimos, las recomendaciones ortotipográficas.
} 
La combinación de los criterios semánticos y sintácticos manejados en este trabajo para el estudio del asturiano permite corroborar la adecuación de la división semántica entre minimizadores partitivos y valorativos (Pinto, 20I5), que además presenta una correspondencia con determinados rasgos sintácticos. Así, los valorativos, que siempre requieren la presencia del determinante indefinido y actúan sobre el predicado verbal, solo son legitimados en contextos antiverídicos, por lo que son términos de polaridad negativa fuertes. Por el contrario, los partitivos presentan un comportamiento más complejo, ya que, acompañados del indefinido, son compatibles con cualquier entorno de polaridad, mientras que como sintagmas nominales escuetos actúan como términos de polaridad negativa fuertes, pues únicamente se legitiman en contextos antiverídicos.

Tras caracterizar brevemente el concepto de minimizador $(\$ \mathrm{I})$ y describir las fuentes utilizadas $\left(\$_{2}\right)$, en el apartado 3 se establece la clasificación semántica de los minimizadores, que se implementará con criterios sintácticos como la sensibilidad a la polaridad, aplicando el modelo de Martins (2000) $(\$ 4)$ y el tipo de cuantificación realizada en los apartados 5 y 6 , respectivamente.

I. Los minimizadores. Definición y criterios de clasificación

Los minimizadores (término acuñado por Bolinger, 1972) son nombres que designan objetos de tamaño mínimo (migaya, gota) o de escaso valor (pimientu, pitu) y "se usan como prototipos de valores mínimos» (RAE-ASALE, 2009, pág. 3678). En virtud de ese contenido semántico se han reanalizado como cuantificadores escalares indefinidos e incluso como refuerzos de la negación oracional.

When these items occur in positive contexts (if they do), they denote a minimal quantity; when they occur in negative contexts, the negation denotes the absence of a minimal quantity, and hence the presence of no quantity at all. This are Bolinger's MINIMIZERS. (Horn, 1989, pág. 400) 
Puesto que constituyen un grupo heterogéneo y su comportamiento no es uniforme, es preciso clasificarlos adecuadamente tanto desde el punto de vista semántico como desde el sintáctico. Conviene aclarar que se descartan en este trabajo los minimizadores que forman modismos o expresiones idiomáticas del tipo no mover un dedo, no decir una palabra, etc. (Sánchez López, 1999) y solo se estudiarán los que tienen libertad combinatoria.

Tanto los datos del asturiano como los de otras lenguas (MeyerLübke, I890-ı906; Jespersen, I917; Bosque, I980; Vallduví, I994; Rueda, I995; Zanuttini, 1997; Medina Granda, 2000; Garzonio y Poletto, 2008, 2009; Pinto, 20I5 y Llop-Naya, 20I7) indican que hay, al menos, cuatro criterios de validez generalizada que permiten explicar las características principales de los minimizadores y establecer grupos diferenciados por su contenido semántico y por su comportamiento sintáctico. La clasificación que propongo parte de una división semántica entre minimizadores partitivos y valorativos (Pinto, 2015), a partir de la cual se irán configurando distintos subgrupos, fundamentalmente en la clase de los partitivos, mediante la aplicación de criterios sintácticos como la sensibilidad a la polaridad negativa, el tipo de cuantificación y la capacidad para funcionar como sintagmas nominales escuetos. La combinación de estas propiedades nos permitirá extraer conclusiones acerca de su comportamiento sintáctico y explicar la aparente falta de sistematicidad que manifiestan estas unidades.

\section{LOS DATOS DEL ASTURIANO}

Los datos utilizados en este trabajo proceden del Atlas Lingüístico de la Península Ibérica $(A L P I)^{2}$ y de Eslema. Corpus Xeneral de la Llingua Asturiana (Eslema).

\footnotetext{
${ }^{2}$ La consulta se ha realizado a partir de la base de datos digitalizada www.alpi.csic.es (García Mouton, 20I6).
} 
El ALPI (Navarro Tomás, s. d.) contiene más de I2o preguntas pensadas para la recogida de información sintáctica, dos de las cuales estaban concebidas para la obtención de expresiones cuantificativas: No tengo ni pizca (ALPI, I, p. 29, 399) y No me importa un pito (ALPI, I, p. 29, 400). Las encuestas en Asturias se realizaron en 23 localidades en los años I932, I934, I935 y 1947, y ofrecen como resultado 95 respuestas y un total de 30 minimizadores distintos. No obstante, los datos se deben tomar con precaución, ya que, aunque la metodología geolingüística es estricta sobre la obligación de evitar respuestas inducidas, es posible que los encuestadores «ofreciesen» algunas opciones a los sujetos informadores (como se colige de algunas de sus anotaciones), lo que habría podido condicionar en cierto modo los resultados. No obstante, esta clase de preguntas, pensadas para obtener expresiones minimizadoras, suele conseguir sin dificultad respuestas espontáneas que contribuyen al estudio del léxico. Sin embargo, para analizar el comportamiento sintáctico de estas unidades los datos del ALPI no solo no son fiables, sino que la rigidez estructural de las respuestas no permite comparar estructuras sintácticas ni detectar microvariación. Por ello, he considerado necesario contrastar y completar los datos con los que ofrece el corpus Eslema.

Eslema (Viejo y Neira, 2008) contiene I700 textos contemporáneos de diversa tipología (literarios, científico-técnicos, periodísticos, etc.) tanto orales como escritos (Viejo et al., 2008), en los que se han podido localizar I2 minimizadores de los recogidos en el $A L P I$ en i63 ejemplos de diversa estructura sintáctica, a los que he añadido dos minimizadores de uso relativamente frecuente en la actualidad: plizcu y duru.

Teniendo en cuenta que el ALPI y Eslema son fuentes de tipología muy diferente y que existe una brecha temporal y sociocultural entre los datos que proporcionan, no es posible hacer una comparación sincrónica rigurosa ni tampoco un estudio de diacronía cercana que permita extraer resultados concluyentes. Sin embargo, sí se pueden observar algunas 


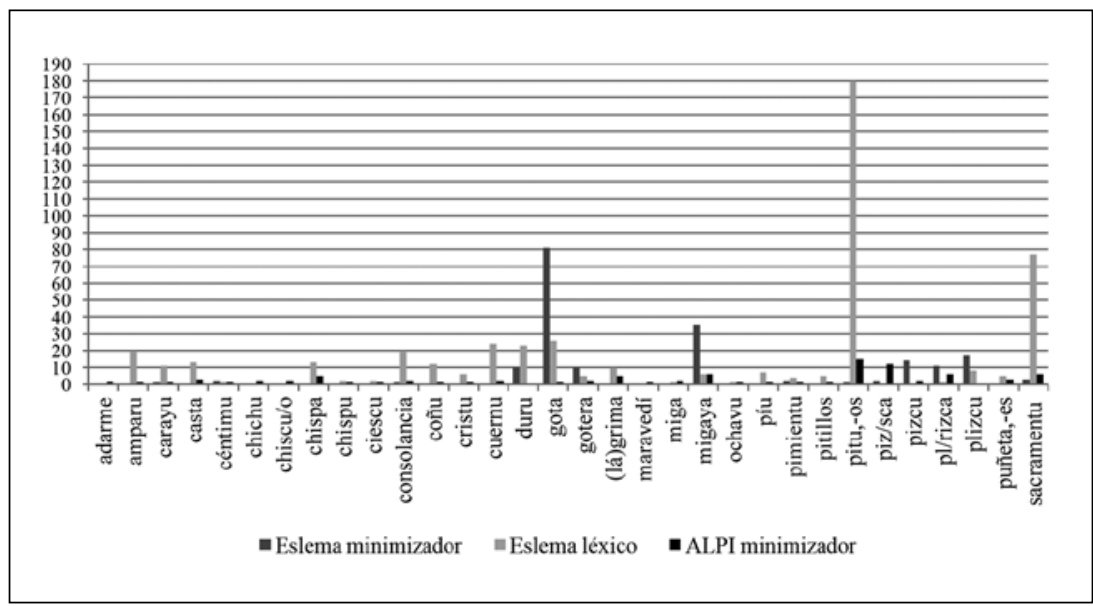

Figura I: Comparativa ALPI-Eslema

tendencias en el comportamiento sintáctico de los minimizadores y en el uso que de ellos hacen los hablantes. Así, el gráfico (Figura I) muestra que varios nombres recogidos en el ALPI no aparecen en Eslema como minimizadores o lo hacen en una frecuencia muy baja, pero sí se registran como unidades léxicas (amparu, casta, chispa, coñu, cuernu, cristu, lágrima, pitu, sacramentu). Resulta llamativo que gota sea el más utilizado de los minimizadores en Eslema y que solo aparezca dos veces en el $A L P I$, pues supondría un proceso de gramaticalización muy rápido, lo que nos lleva a pensar en la posibilidad de que los encuestadores dirigiesen las respuestas hacia resultados castellanos, entre los que no figura gota. En cualquier caso, hay que ser cautos ante los resultados de la comparación, ya que el hecho de que algunos minimizadores no aparezcan en Eslema no significa que hayan desaparecido, del mismo modo que tampoco tenemos certeza de que todos los que aparecen en el ALPI estuviesen realmente vivos en aquella época. 
Para el cómputo no se ha tenido en cuenta la variación fonética de algunas unidades (plizca / prizca, pizca / pisca, chiscu / chisco, lágrima / grima), ni las diferencias en el uso de singular o plural (puñeta / puñetes, pitu / pitos). Sin embargo, la variación de género sí se ha considerado un criterio diferenciador para computar las formas femeninas y las masculinas por separado, ya que resulta significativo para la distribución sintáctica y semántica del minimizador así como para determinar su grado de gramaticalización.

\section{Clasificación semántica: \\ MiNIMIZADORES VALORATIVOS Y PARTITIVOS}

Siguiendo a Pinto (20I5, pág. II2), desde el punto de vista léxico, podemos distinguir dos grandes grupos: los minimizadores partitivos, que son "todos aqueles que tiveram origem em nomes comuns com propriedades escalares que designam a parte mais pequena de um todo» (una gota, una migaya); y los minimizadores valorativos, que "provêm de nomes comuns que denotam um valor irrisório ou pequena dimensão» (un pimientu, un cuernu). La clasificación resultante para el asturiano sería la que se muestra en la tabla I.

\begin{tabular}{lll|ll}
\hline \multicolumn{3}{c|}{ Valorativos } & \multicolumn{2}{c}{ PARTitivos } \\
\hline insignificantes & vulgares & monedas & chispa & migaya \\
amparu & carayu & adarme & chispu & piz/sca \\
casta & ciescu & céntimu & consolancia & pizcu \\
chichu & coñu & duru & gota & pl/rizca \\
chiscu/o & cristu & maravedí & gotera & plizcu \\
cuernu & peu/ píu & ochavu & (lá)grima & sacramentu \\
pimientu & pitu,-os & & miga & \\
pitillos & puñeta,-es & & \\
\hline
\end{tabular}

TABla I: Minimizadores. Clasificación semántica 
Dentro de los valorativos se pueden hacer tres subgrupos: los que designan objetos insignificantes, los que nombran unidades monetarias y los vulgares (Postal, 2004), que hacen referencia a expresiones malsonantes o tabúes de tipo sexual, fisiológico o religioso (un carayu, un coñu, un peu, un cristu). Todos ellos tienen en común que siempre van acompañados del determinante indefinido, actúan sobre el predicado verbal y solo aparecen en contextos negativos, por lo que, como veremos en el siguiente apartado, se comportan siempre como términos de polaridad negativa. No obstante, el $A L P I$ registra algunas excepciones que se reflejan en (I)-(5). Los dos casos de casta se recogen en Cimiano y podrían deberse al carácter abstracto del nombre. Los otros tres contienen nombres de monedas y proceden del mismo punto de encuesta, Sames, donde todas las respuestas recogidas tienen la misma estructura (con el verbo tener) y no llevan determinante, por lo que parece que se buscaba más la variedad de vocablos que su uso sintáctico.

(I) A mí nun me importa ni casta (322, Cimiano)

(2) Nun tengo ni casta (322, Cimiano)

(3) Nun tengo céntimu (320, Sames)

(4) Nun tengo maravedí (320, Sames)

(5) Nun tengo amparu (320, Sames)

En el caso de los minimizadores valorativos, no es extraño que algunos nombres hayan desaparecido como minimizadores y se mantengan como unidades léxicas (vid. Figura I), pues como apunta Rueda (1995), este tipo de expresiones están estrechamente vinculadas con los prototipos de valor mínimo de cada época, por lo que su inventario se modifica con más frecuencia que el de los partitivos. Esto ocurre especialmente con los vulgares, como pitu, cuya polisemia lo convierte en un nombre de uso habitual con cualquiera de sus significados; pero, sin embargo, apenas se utiliza como minimizador vulgar, probablemente porque ha 
sido sustituido en estos contextos por otro tipo de palabras con connotaciones vulgares semejantes.

Los partitivos, por el contrario, si bien son más uniformes desde el punto de vista semántico, pues denotan porciones de un todo, presentan un comportamiento sintáctico más complejo, ya que no solo puede variar el elemento sobre el que se realiza la cuantificación, sino que la ausencia del determinante en entornos de polaridad negativa altera la estructura sintáctica y pone de manifiesto el reanálisis del minimizador como cuantificador o como marcador negativo enfático. A estas cuestiones dedicaré los apartados siguientes.

\section{Clasificación sintáctica i. \\ SENSIBILIDAD A LA POLARIDAD NEGATIVA}

La polaridad es una relación de dependencia semántica que se establece entre una unidad que legitima o induce dicha relación (legitimador o inductor) y una unidad dependiente, que se denomina término de polaridad (Baker, 1970). Los términos de polaridad se clasifican, por tanto, en función del tipo de contexto que los legitima (Giannakidou, 1997). Así, son términos de polaridad positiva (TPP) los que son legitimados en entornos verídicos o positivos, en oposición a los términos de polaridad negativa (TPN), que dependen semánticamente de un inductor antiverídico o negativo, y los términos de polaridad modal (TPM), legitimados por un inductor no verídico o modal (modos verbales, predicados inherentemente intensionales o negativos, construcciones genéricas (Bosque, 1999).

Los trabajos sobre la polaridad y sus efectos en la cuantificación en español (Bosque, I980; Sánchez López, I999; González-Rodríguez, 2008) y en catalán (Espinal, 2008; Llop-Naya, 2017), coinciden en considerar que son TPN tanto los que aparecen en contextos no verídicos en general como los que aparecen específicamente en entornos antiverídicos. 
Por lo tanto, existiría una oposición entre I) contextos verídicos, que legitiman TPP, y 2) contextos no verídicos, que legitiman TPN, dentro de los cuales es posible distinguir entre términos de polaridad negativa débiles (TPND) (legitimados en contextos no verídicos y antiverídicos) y términos de polaridad negativa fuertes (TPNF) (legitimados en contextos antiverídicos).

Sin embargo, los efectos de la polaridad sobre los cuantificadores indefinidos (entre los que se incluyen los minimizadores) en asturiano muestra una situación distinta, que se aproxima más al modelo portugués (Martins, 2000), pues los contextos no verídicos no legitiman la presencia de indefinidos negativos; únicamente pueden aparecer en estos entornos los indefinidos positivos. La propuesta de Martins consiste en una clasificación de los indefinidos a partir de la combinación de tres rasgos asociados a la polaridad: negación [neg], modalidad [mod] y afirmación [aff], que se corresponden con los tres tipos de entornos ya citados (antiverídicos, no verídicos y verídicos, respectivamente), con tres posibles valores: positivo $[+]$, negativo $[-]$, sin especificar $[\alpha]$.

I hypothesize that the grammatical encoding or polarity [...] together with semantic considerations $[\ldots]$ derive the fact that strong polarity items have at most one specified feature value. On the other hand, following the same line of reasoning, weak polarity items will have at least one polar feature with a variable underspecified $(\alpha)$ value. (Martins, 2000: págs. 209-210)

A diferencia de otras lenguas románicas, en portugués europeo actual y en asturiano los TPN son TPNF, pues solo están especificados para el rasgo negación, por lo que únicamente se legitiman en los entornos antiverídicos 3 : [o-aff, +neg, o-mod]. Por su parte, los indefinidos positivos pueden aparecer tanto en entornos no verídicos como en entornos

3 Subsisten algunas expresiones fosilizadas de periodos anteriores como ¿Qué sabe nadie? Sobre los cambios en el paradigma de los indefinidos en las lenguas románicas, véase Martins (2000). 
verídicos, lo que Martins (2000) atribuye a la no especificación de los rasgos [aff] y [mod], que los convierte en términos de polaridad positiva débiles (TPPD): [ $\alpha$-aff, o-neg, $\alpha$-mod]. Es decir, en el caso del portugués y del asturiano, la oposición se produce entre I) contextos antiverídicos (que inducen la presencia de TPNF (6)) y 2) contextos no antiverídicos o positivos, donde se legitiman los términos de polaridad modal y positiva, que pueden considerarse TPPD (7).

(6) TPNF [o-aff, +neg, o-mod]
a. $\quad *$ Llamó dalu/ naide
(verídico)
b. *Si llama dalu/ naide, avísesme
(no verídico)
c. Nun llamó dalu/ naide
(antiverídico)

(7) TPPD [ $\alpha$-aff, o-neg, $\alpha$-mod]
a. Llamó daquién/ algunu
(verídico)
b. Si llama daquién/ algunu, di que nun toi
(no verídico)
c. *Nun llamó daquién/ algunu
(antiverídico)

Los minimizadores partitivos, cuando van acompañados del determinante indefinido (8), (9), no son sensibles a la polaridad, ya que pueden aparecer en cualquiera de los tres contextos, por lo que pueden considerarse términos bipolares ${ }^{4}$ (TB), cuya característica es la inespecificación para los tres rasgos [ $\alpha$-aff, $\alpha$-neg, $\alpha$-mod]. En cambio, si se utilizan como singulares escuetos (sin determinante) solo son gramaticales como TPNF [o-aff, +neg, o-mod] (Io), pues únicamente bajo el alcance de la negación se reanalizan como cuantificadores o marcadores negativos enfáticos. Por su parte, los minimizadores valorativos (II), que siempre van precedidos del indefinido, son TPNF porque solo resultan legitimados por la presencia de la negación.

(8) Minimizadores TB [ $\alpha$-aff, $\alpha$-neg, $\alpha$-mod]: partitivos

${ }^{4}$ Etiqueta utilizada por Martins (2000), quien la toma de Van der Wouden (1994). 
a. Movíme una gota y despertélu (verídico)

b. Si pudieras movete una gota cabíamos toos en bancu (no verídico)

c. Nun me moví una gota mientres m'escayolaben (antiverídico)

(9) Minimizadores TB [ $\alpha$-aff, $\alpha$-neg, $\alpha$-mod]: partitivos

a. Comí un plizcu chorizu (verídico)

b. Si pudieras dame un plizcu chorizu, agradecíatelo bien. (no verídico)

c. Nun comí un plizcu chorizu (antiverídico)

(Io) Minimizadores TPNF [o-aff, +neg, o-mod]: partitivos escuetos

a. *Movíme gota y despertélu (verídico)

b. *Si pudieras movete gota, cabíamos toos en bancu (no verídico)

c. Nun me moví gota pa nun despertalu (antiverídico)

(II) Minimizadores TPNF [o-aff, +neg, o-mod]: valorativos
a. $\quad *$ Eso val un pimientu
(verídico)
b. *Eso valdrá un pimientu
(no verídico)
c. Eso nun val un pimientu
(antiverídico)

Por lo tanto, una primera clasificación sintáctica basada en la polaridad se corresponde con la semántica, tal como se muestra en la tabla 2.

\begin{tabular}{|c|c|c|c|c|}
\cline { 3 - 4 } \multicolumn{2}{c}{} & \multicolumn{2}{c|}{ Clasificación semántica } \\
\cline { 3 - 5 } & & partitivos & valorativos \\
\hline \multirow{2}{*}{$\begin{array}{c}\text { Clasificación } \\
\text { sintáctica }\end{array}$} & polaridad & TB & $+\mathrm{D}$ & \\
\cline { 3 - 5 } & TPNF & $-\mathrm{D}$ & $+\mathrm{D}$ \\
\hline
\end{tabular}

TABla 2: Minimizadores Clasificación sintáctica I

\section{Clasificación sintáctica 2. \\ Ámbito de LA CUANTIFICACiÓN ESCALAR}

Los minimizadores, puesto que designan objetos de pequeño tamaño o escaso valor, comportan un valor escalar que los sitúa en la posición 
más baja de una escala semántica o pragmática, lo que genera implicaturas cuando se hallan bajo el alcance de la negación. Si aplicamos el Principio escalar de Fauconnier (I2), negar el extremo inferior de una escala implica negar la escala completa.

(I2) If $\mathrm{x} 2$ is lower than $\mathrm{xI}$ on the scale $\mathrm{S}$ associated with $\mathrm{R}(\mathrm{x}, \ldots)$, then $\mathrm{R}(\mathrm{x} 2, \ldots)$ implies $\mathrm{R}(\mathrm{xI}, \ldots)$; thus, in particular if $\mathrm{R}$ holds for the lowest element on $\mathrm{S}$, it holds for all elements of $S$ (call this the scale principle). (Fauconnier, 1975: pág. 362)

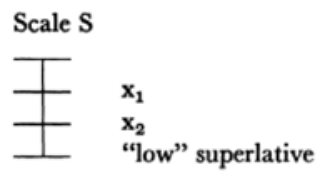

Sin embargo, en los contextos positivos, la afirmación de un extremo de la escala no implica la afirmación de los puntos superiores. Para dar cuenta de esta doble interpretación de los minimizadores, Llop-Naya (20I7) aplica al catalán la propuesta de Chierchia (2006) y considera que los minimizadores pueden tener dos variantes asociadas al rasgo escalar $[ \pm \sigma]$. Así, en los contextos positivos, el minimizador tendría el rasgo [- $\sigma]$, que mantiene inactivos los otros puntos de la escala; mientras que en los contextos negativos, donde está activa toda la escala, el minimizador tendría el rasgo $[+\sigma]$.

Las dos variantes $[ \pm \sigma]$ están presentes únicamente en los minimizadores partitivos (I3)-(I5), como veremos, pues los valorativos, (I6) y (I7), como son TPNF y solo aparecen en contextos negativos, siempre mantienen activos todos los puntos de una escala cualitativa, luego son $[-\sigma]$. Este tipo de minimizadores actúan a nivel oracional para expresar que algo no cumple con el grado mínimo exigible. Por eso suelen aparecer con verbos de estimación o de precio (valir 'valer', emportar 'importar'). 
Los minimizadores vulgares ( 17 ) presentan el mismo comportamiento, pero, como explica Postal (2004) pueden expresar minimalidad con respecto a distintas dimensiones, pues no presentan las restricciones semánticas de los valorativos no vulgares.

(13) Charramos una migaya y dormimos $[-\sigma]$

(I4) Nun se mueva una migaya (=nada) $[+\sigma]$

(is) ... nun tenía talante de negociador, esixía ensin ceder migaya [- $\sigma]$

(I6) Nun m’importa un pimientu (ALPI, 310, Sames) [-б]

(17) Nun m’importa un pitu (ALPI, 315, Felechosa) [- $\sigma]$

Por su parte, los minimizadores partitivos, en principio, no se muestran sensibles a la polaridad, sino que son bipolares (TB), aunque, al adquirir autonomía funcional y prescindir del indefinido (I5), se convierten en TPNF [- $\sigma]$. Puesto que denotan partes o porciones de un todo, estos minimizadores pueden actuar como cuantificadores de un nombre continuo o bien como cuantificadores del predicado verbal a nivel oracional. Como explico en otros trabajos (San-Segundo-Cachero, 2017, aceptado), y como se ha demostrado en otras lenguas románicas (Roberts y Roussou, 2003; Garzonio y Poletto, 2008, 2009; Llop-Naya, 2017), el uso del minimizador como cuantificador oracional es consecuencia de un proceso de gramaticalización que permite su extracción de un sintagma nominal que funcionaría como OD, (I8) y (19). Me ocuparé de estas cuestiones en el siguiente apartado. Por ahora, atendiendo al tipo de cuantificación que se efectúa, podemos implementar la clasificación de los minimizadores tal como se refleja en la tabla 3.

(18) Nun tenía un plizcu de potencia muscular

(Homes)

(19) ensin plizca enfotu nes idees universalistes

(Diálogu) 


\begin{tabular}{|c|c|c|c|c|}
\hline & & & \multicolumn{2}{|c|}{ Clasificación semántica } \\
\hline & & & partitivos & valorativos \\
\hline \multirow{4}{*}{$\begin{array}{l}\text { Clasificación } \\
\text { sintáctica }\end{array}$} & \multirow{2}{*}{ polaridad } & $\mathrm{TB}$ & $+\mathrm{D}$ & \\
\hline & & TPNF & $-\mathrm{D}$ & $+\mathrm{D}$ \\
\hline & \multirow{2}{*}{ cuantificación } & nominal & $\pm \mathrm{D}$ & \\
\hline & & verbal & $\pm \mathrm{D}$ & $+\mathrm{D}$ \\
\hline
\end{tabular}

TABla 3: Minimizadores. Clasificación sintáctica 2

\section{SUbCLASIFICACIÓN DE LOS MINIMIZAdORES PARTITIVOS: CUANTIFICACIÓN Y DETERMINACIÓN}

Puesto que los minimizadores partitivos pueden actuar como modificadores del verbo $(\mathrm{Mv})$ o modificadores de otro nombre $(\mathrm{Mn})$ con el que forman una construcción (pseudo)partitiva y estas opciones combinatorias reflejan diferencias en lo que respecta a la polaridad, la primera subdivisión de los minimizadores partitivos se realizará según el tipo de cuantificación que efectúan. Utilizaré únicamente los datos de Eslema, ya que los que proporciona el $A L P I$, por su rigidez sintáctica, no permiten detectar variación en la estructura sintáctica.

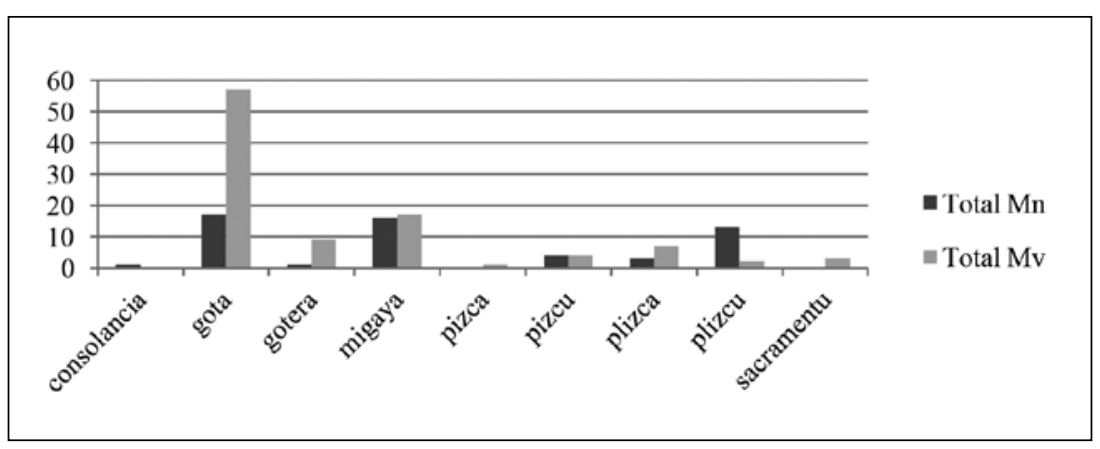

Figura 2: Eslema. Minimizadores Mn y Mv 
Como se puede ver en el anterior gráfico (Figura 2), el uso y la distribución de los minimizadores son muy heterogéneos: gota es el más utilizado como modificador verbal, mientras que las cifras de su uso en construcciones nominales (pseudo)partitivas se asemejan a las de migaya, cuya presencia en ambos contextos es muy similar.

\section{I. Cuantificación nominal}

Dentro del SN los minimizadores actúan como nombres cuantificativos en una construcción partitiva o pseudopartitiva. Estas construcciones son estructuras bimembres que constan de una cabeza y una coda articuladas por la preposición de (Milner, 1978; Brucart; 1997; Sánchez López, I999; Alexiadou et al., 2007; RAE-ASALE, 2009; Martí, 20IO; Demonte y Pérez-Jiménez, 20I5). Si la coda designa un conjunto extensionalmente determinado (sintagma determinante o SD), la construcción resultante es de tipo partitivo, pues el minimizador acota una porción de una sustancia; mientras que, cuando la coda es un conjunto indeterminado o sintagma nominal ( $\mathrm{SN}$ ), la construcción que se genera es pseudopartitiva o cuantitativa. Obsérvese que, aunque en el nivel intrasintagmático la preposición de puede omitirse en asturiano siempre que la palabra precedente no acabe en consonante (ALLA, 1999), en este tipo de construcciones solo es posible si la coda es un SN (20), pues las partitivas exigen siempre la preposición (2I). La presencia / ausencia de la preposición en estos casos es un fenómeno sintáctico-semántico que debe ser estudiado con detalle, ya que puede haber favorecido la gramaticalización de los minimizadores partitivos, como se explica en San Segundo Cachero (aceptado), pero no tiene especial relevancia en este trabajo donde los minimizadores se estudian desde una perspectiva sincrónica.

(20) Pseudopartitivas

a. una gota (de) vinu

b. una gota (de) vergoña 

c. una migaya (de) chorizu
d. una migaya (de) vergoña

(2I) Partitivas
a. una gota del vinu/ *una gota el vinu
b. una migaya del chorizu/*una migaya el chorizu

La sensibilidad de los minimizadores a la polaridad está condicionada por la presencia del determinante indefinido, como se refleja en el gráfico (Figura 3). Los minimizadores acompañados del indefinido $(\mathrm{Mn}+\mathrm{D})$ son términos bipolares (TB) o inespecíficos [ $\alpha$-aff, $\alpha$-neg, $\alpha$-mod], compatibles con cualquier entorno de polaridad. No obstante, en los ejemplos de Eslema los legitimadores son contextos verídicos y antiverídicos, que se representan en el gráfico como TB-V y TB-A, respectivamente. En cambio, solo los contextos antiverídicos legitiman el minimizador escueto (Mn-D) como cabeza de la construcción (pseudo)partitiva, que sería un TPNF [o-aff, +neg, o-mod], pues un singular escueto no es gramatical a menos que se reanalice como una categoría semiléxica o funcional sobre la que han dejado de operar las restricciones nominales.

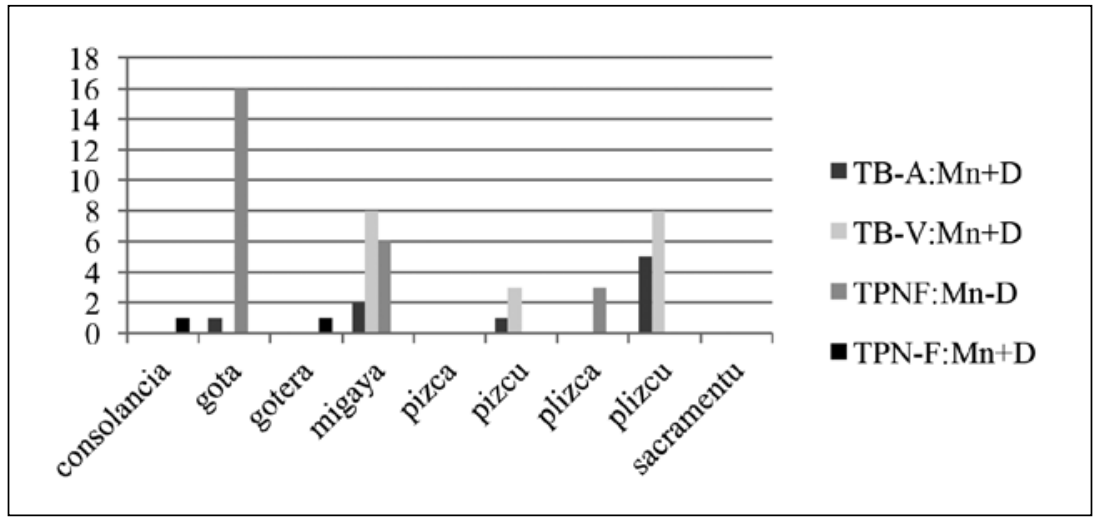

Figura 3: Eslema. Modificadores nominales 
Por lo tanto, mientras el minimizador va acompañado del indefinido, se interpreta como una expresión cuantificativa nominal (SQ) totalmente gramatical en cualquier contexto.

El gráfico muestra que consolancia, gotera, pizcu y plizcu no prescinden del determinante indefinido; migaya es el único registrado en las tres posibilidades contextuales; mientras que gota (22), el más utilizado sin determinante y, por tanto, el más gramaticalizado, no se registra en entornos verídicos, al igual que plizca (23), que solo aparece en el corpus como minimizador escueto. En estos casos el minimizador puede interpretarse como un cuantificador o como un refuerzo de la negación oracional similar a en absoluto. No obstante, en la lengua oral tanto gota como plizca aparecen en entornos positivos acompañados del determinante indefinido.

(22) Nun me costó gota trabayu

(Románticu)

(23) ... ensin plizca enfotu nes idees universalistes

(Diálogu)

(24) Non perdí ni una migaya del to cuentu

(Cuentiquinos)

(25) ... pa que nun entre una gotera d'aire

(Pisu)

Por lo tanto, los $\mathrm{Mn}+\mathrm{D}$ que pueden considerarse polarmente inespecíficos o bipolares (TB) serían: una migaya, un pizcu y un plizcu, según datos de Eslema, a los que habría que añadir una gota y una plizca. Los que quedan restringidos a los entornos negativos serían una consolancia y una gotera y, por prescindir del determinante, gota, migaya y plizca, por lo que pueden considerarse TNPF fuertes.

La diferencia entre los TPNF con determinante y sin determinante radica en el grado de gramaticalización del minimizador: los más gramaticalizados pueden prescindir de él porque se han reanalizado como cuantificadores dentro de la construcción (pseudo)partitivas.

5 Roberts y Roussou, 2003; Garzonio y Poletto, 2008; Llop-Naya, 2017, entre otros, explican el reanálisis $(\mathrm{N}$-to-Num) mediante un modelo monoproyeccional, mientras que San-Segundo-Cachero 


\subsection{Cuantificación verbal}

El valor cuantificativo escalar que retienen los minimizadores una vez despojados de sus rasgos nominales, les permite actuar como constituyentes oracionales capaces de modificar el predicado verbal, por lo que se los puede considerar adverbios cuantificadores o adverbios minimizadores (26)-(28). Su inserción en la estructura oracional supone un paso más en el proceso de gramaticalización, favorecido por los contextos ambiguos en los que el minimizador puede interpretarse como argumento o como cuantificador (Llop-Naya, 20I7, págs. 154-160 y las referencias allí indicadas).

(26) ... pensaba qu'ensin comer migaya en tres díes nun diba tener fuercies

(Potestas)

(27) Basil nun prebó gota.

(Home)

(28) ... final nun comen un sacramentu

(Escaeceme)

El uso adverbial de los minimizadores como modificadores verbales (Mv) es claramente mayoritario en los contextos negativos (3I)-(35), donde el rasgo escalar $[+\sigma]$ activa todos los puntos de la escala (Chierchia, 2006). En los entornos no negativos el minimizador siempre tiene que ir acompañado del determinante, (29) y (30), puesto que los singulares escuetos resultan agramaticales, y equivale a un poco. En cambio, en los entornos de polaridad negativa de nuevo la ausencia del indefinido (33)(35), nos indica un cambio en el estatus gramatical del minimizador que genera una interpretación equivalente a nada (lectura cuantitativa) o en absoluto (lectura negativa enfática).

\footnotetext{
(aceptado) explica las construcciones (pseudo)partitivas basándose en la hipótesis de la predicación invertida (Kayne, 1994; Corver, 1998; Martí, 2010) y considera que el reanálisis N-to-Num se produce en la expresión cuantificativa o SQ y no en la expresión nominal en conjunto.
} 
(29) Charramos una migaya y dormimos

(30) ... aquél que las espurra una plizca, ¡zas!

(3I) ... ya como nun oigo una gota

(32) Nun se mueva una migaya

(33) Y nun esaxero gota

(34) ... nun me prestó pizca

(35) ... nun callaba gotera
(L'Acampallengua)

(Tiempos)

(Escaecéme)

(Psicokiller)

(L'Andecha)

(L'arna)

(L'arna)

La presencia de minimizadores adverbiales parece estar condicionada por el aspecto semántico del predicado, ya que por su valor escalar solo cuantifican predicados que puedan proyectarse en una escala y que puedan ser divididos en eventos sucesivos menores. Garzonio y Poletto (2008, pág. I6) explican que estas restricciones están presentes en el uso del cuantificador italiano gnente y se derivan de su carácter escalar monotónico decreciente: «the property of requiring a set of discrete events comes from the monotonic property and the fact that they have to be ordered onto a strictly decreasing scale comes from it being a strictly decreasing quantifier». Como consecuencia del valor escalar, gnente es compatible con predicados susceptibles de ser cuantificados escalarmente, pero la escala tiene que ser temporal, ya que un determinado tipo de suceso o evento no puede descomponerse en partes o en grados.

Los únicos predicados que admiten cuantificación escalar monotónica son los atélicos, lo que explica que gnente (36) y los minimizadores asturianos (37) no aparezcan si el predicado selecciona un OD referencial $^{6}$. En el caso del asturiano es posible interpretar el minimizador como cuantificador equivalente a nada o bien como partícula enfática negativa.

${ }^{6}$ Obsérvese que sí sería posible Nun ve / mira una gota la tele, porque en este caso la tele no tiene valor referencial, no se refiere a un objeto concreto. La expresión ver / mirar la tele constituye locución verbal que designa una actividad, por lo que sí sería cuantificable y, por tanto, compatible un minimizador escalar. 
(36) a. *Nol me leze gnente i libri, sto fio

b. Nol me leze gnente libri, sto fio (actividad: leer libros)

c. Nol dorme gnente

d. Nol lavora gnente

（37） a. *Nun me llee una gota los llibros esti neñu

b. Nun me llee una gota llibros esti neñu (actividad: leer libros)

c. Nun duerme una gota (nada/ en absoluto)

d. Nun trabaya una gota (nada/ en absoluto)

En el siguiente gráfico (Figura 4) podemos ver que una migaya, un pizcu, una plizca y un plizcu son TB, puesto que quedan legitimados en contextos verídicos y antiverídicos. Por el contrario, son TPNF una gota, un sacramentu y todos los que pueden aparecer como minimizadores escuetos: gota (el más utilizado), gotera, migaya, pizca y sacramentu.

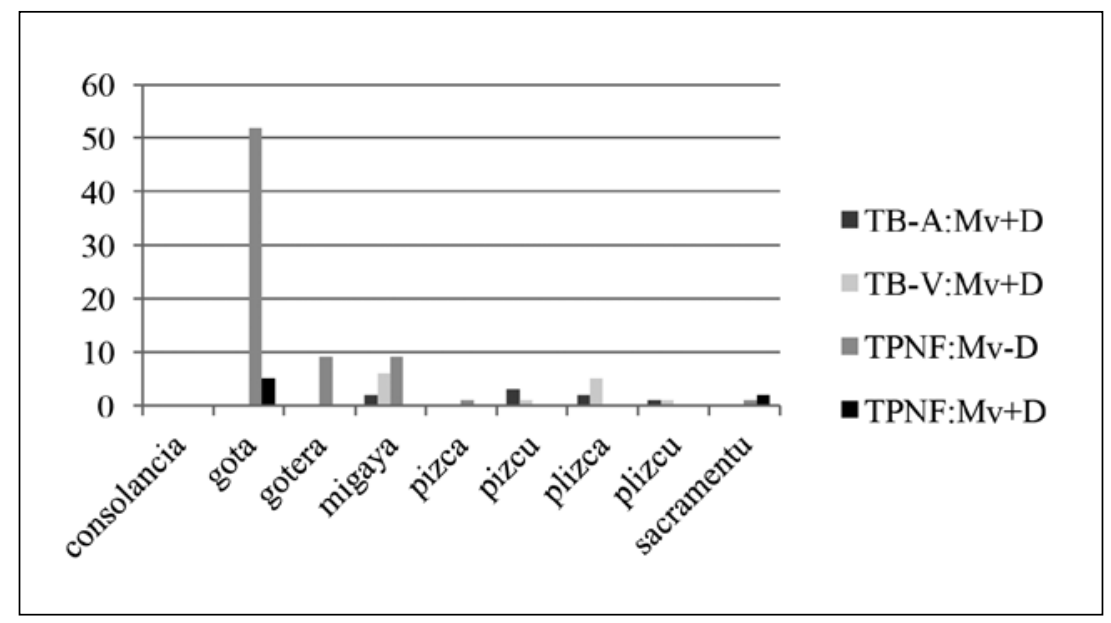

Figura 4: Eslema. Modificadores verbales 


\subsection{Cuantificación adjetival y adverbial}

Por último, hay un grupo de minimizadores partitivos que, acompañados del determinante indefinido, adoptan un valor adverbial que les permite modificar a otros adverbios (4I)-(43) o a adjetivos (38)-(40). Todos los casos que se registran en Eslema (Figura 5) aparecen en contextos positivos y aportan un valor de pequeña cantidad (un poco); sin embargo, también pueden utilizarse con este mismo valor en contextos negativos. De nuevo, se echa en falta en el corpus la presencia de una gota en esta función de modificador intrasintagmático, tan habitual en el uso oral.

(38) La moza volvióse un pizcu despistada buscando al autor de les voces.

(Estatutos)

(39) ... con fileres d'árboles que dalgún edil un pizcu currutacu bautizara pomposamente col nome del Bulevar.

(Estatutos)

(40) ... pámique tenía d'andar una migaya tresmanada

(Bodes)

(4I) ... al querer arrimala un pizcu más a él,

(Estatutos)

(42) ... ya una plizca detrás, la caliza silueta del Cornón

(Tiempos)

(43) ... ¿qué tienen les fiestes de Navidá que tolos famosos tienen que facer el tontu un plizcu más de lo avezao?

(Nomes)

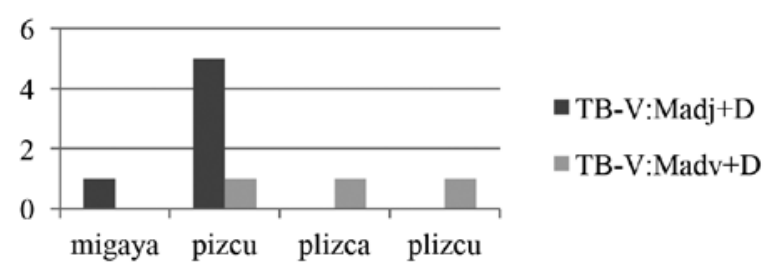

Figura 5: Eslema. Modificadores Madj y Madv 


\section{Conclusiones}

La combinación de los criterios semánticos y sintácticos manejados en este trabajo permite corroborar la adecuación de la división semántica entre minimizadores partitivos y valorativos, que además presenta una correspondencia con determinados rasgos sintácticos. Los valorativos requieren siempre la presencia del indefinido, actúan sobre el predicado verbal y solo aparecen en entornos antiverídicos, por lo que son TPNF.

Por el contrario, los partitivos tienen un comportamiento sintáctico más complejo. La ausencia del indefinido indica que el minimizador se ha gramaticalizado y no está sujeto a las restricciones nominales, fenómeno que solo se produce en los contextos antiverídicos, por lo que los minimizadores escuetos son TPNF, tanto si actúan sobre un nombre $(\mathrm{Mn})$ como si lo hacen sobre el predicado verbal $(\mathrm{Mv})$. La interpretación del minimizador escueto como un constituyente oracional hace posible su reanálisis como marcador negativo enfático, especialmente cuando en la oración hay un OD referencial que bloquea automáticamente la cuantificación monotónica decreciente que se suscita con verbos atélicos y que genera ambigüedad.

Acompañados del indefinido, los minimizadores partitivos son TB, por lo que no se ven restringidos por la polaridad oracional. Así, en los contextos positivos indican una pequeña cantidad, el punto más bajo en la escala, pero no mantienen activos los otros puntos de la escala porque presentan el rasgo $[-\sigma]$, que impide la generación implicaturas escalares. En cambio, en los contextos negativos, el rasgo escalar tiene valor positivo $[+\sigma]$ de modo que toda la escala está activa y se produce un enriquecimiento del significado mediante mecanismos inferenciales, por lo que el minimizador resulta ambiguo entre una lectura cuantitativa equivalente a nada o una lectura negativa enfática. 
La clasificación de los indefinidos según la sensibilidad a los distintos entornos de polaridad propuesta por Martins (2000) permite establecer un paralelismo entre el asturiano y el portugués y al mismo tiempo diferenciar estas lenguas de los otros romances peninsulares, pues en ambas los indefinidos negativos se legitiman únicamente en entornos antiverídicos, por lo que son TPNF. En cambio, los entornos no verídicos y verídicos pueden legitimar indefinidos positivos, que resultan ser TPPD. Esta combinación de rasgos es aplicable a los minimizadores, ya que en función de su contenido semántico y de la capacidad de funcionar como $\mathrm{SN}$ escuetos exhiben un comportamiento diferente, ya sea como TNPF, ya sea como TB, insensibles a la polaridad.

En la siguiente tabla se recoge la clasificación de los minimizadores realizada a partir de los datos de Eslema y del $A L P I$, combinando criterios sintácticos y semánticos.

\begin{tabular}{|c|c|c|c|c|}
\hline & \multicolumn{3}{|c|}{ Clasificación sintáctica } \\
\hline & & Polaridad & Determinante & Cuantificación \\
\hline \multirow{4}{*}{$\begin{array}{l}\text { Clasificación } \\
\text { semántica }\end{array}$} & \multirow[t]{2}{*}{ Partitivos } & $\mathrm{TB}$ & $+\mathrm{D}$ & $\begin{array}{l}\text { Verbal, nominal, } \\
\text { adjetival, adverbial }\end{array}$ \\
\hline & & TPNF & $\pm \mathrm{D}$ & Verbal, nominal \\
\hline & \multirow{2}{*}{ Valorativos } & $\mathrm{TB}$ & & \\
\hline & & TPNF & $+\mathrm{D}$ & Verbal \\
\hline
\end{tabular}

Tabla 7: Minimizadores. Clasificación sintáctico-semántica

\section{REFERENCIAS BIBLIOGRÁFICAS}

Alla (1999) = Academia de la Llingua Asturiana, Gramática de la llingua asturiana, Uviéu (Academia de la Llingua Asturiana), 1999 [3. $\left.{ }^{\mathrm{a}} \mathrm{ed} .200 \mathrm{I}\right]$.

Alexiadou et al. (2007) = Artemis Alexiadou, Liliane Haegeman \& Melita Stavrou, Noun phrase in the generative perspective, Berlin (Walter de Gruyter), 2007. 
Baker (1970) = Carl Lee Baker, "Double negatives», Linguistic Inquiry, I, 2 (1970), págs. 169-86.

Bolinger (1972) = Dwight Bolinger, Degree words, La Haya (Mouton), 1972.

Bosque (1980) = Ignacio Bosque, Sobre la negación, Madrid (Cátedra), 1980.

BosQue (1999) = IgNaCio BosQue, «Sobre la gramática de los contextos modales. Entornos modales y expresiones inespecíficas en español», en J. A. SAmper Padilla \& M. Troya Déniz (coords.), Actas del XI Congreso Internacional de la Asociación de Lingüistica y Filología de la América Latina, Las Palmas (Universidad de Las Palmas de Gran Canaria), 1999, págs. 43-58.

BRUCART (1997) = José MARIA BRUCART, "Concordancia ad sensum y partitividad en español», en M. Almeida \& J. Dorta (eds.), Contribuciones al estudio de la lingüisstica hispánica. Homenaje al profesor Ramón Trujillo, Barcelona (Montesinos), 1997, págs. I57-I84.

Chierchia (2006) = Gennaro Chierchia, "Implicatures of domain widening», Linguistic Inquiry, 37, 4 (2006), págs. 535-590.

Corver (1998) = Norbert Corver, «Predicate movement in pseudopartitive constructions", en A. Alexiadou \& C. Wilder (eds.), Possessors, predicates and movement in the determiner phrase, Amsterdam (Jonh Benjamins), 1998, págs. 215-257.

Demonte \& Pérez-Jiménez (20I5) = Violeta Demonte \& Isabel Pérez-JimeNEZ, "Construcciones partitivas y pseudopartitivas en español: concordancia híbrida y variación en la interficie sintaxis-semántica», en E. Hernández \& P. Martín BUTRAGUEÑo (eds.), Variación y diversidad lingüistica. Estudios en busca de una teoría convergente, México (El Colegio de México), 2015, págs. I5-98.

Espinal (2008) = Maria Teresa Espinal, «La negació», en J. Solà, M. R. Lloret, J. Mascró \& M. Pérez Saldanya (dirs.), Gramàtica del català contemporani, vol. 3, Barcelona (Empúries), 2008, págs. 2729-2793.

Fauconnier (1975) = Gilles Fauconnier, «Pragmatic scales and logical structure», Linguistic Inquiry, 6, 3 (1975), págs. 353-375.

García Mouton (20i6) = Pilar García Mouton (coord.), Inés FernándezOrdóñez, David Heap, Maria Pilar Perea, João Saramago \& Xulio Sousa, ALPICSIC [www.alpi.csic.es], edición digital de Tomás Navarro Tomás (dir.), Atlas Lingüistico de la Peninsula Ibérica, Madrid (CSIC), 2016.

Garzonio \& Poletto (2008) = Jacopo Garzonio \& Cecilia Poletto, «Minimizers and quantifiers: a window on the development of negative markers», Studies in Linguistics, Working Papers, 2 (2008), págs. 59-80. 
Garzonio \& Poletto (2009) = Jacopo Garzonio \& Cecilia Poletto, «Quantifiers as negative markers in Italian dialects», University of Venice Working Papers in Linguistics, I9 (2009), págs. 8I-I09.

GonzÁlez-Rodríguez (2008) = Raquel GonzÁlez-Rodríguez, La Polaridad positiva en español. Tesis doctoral. Universidad Complutense, 2008, http://eprints. ucm.es/8I45/.

Horn (1989) = LaURenCE Horn, A natural history of negation, Standford (CSLI Publications), 1989 [200I].

Jespersen (I9I7) = OtTo Jespersen, Negation in English and other languages, Copenhague (A. F. Host \& son), I9I7.

Kayne (1994) = Richard Kayne, The antisymmetry of syntax, Cambridge, M. A. (MIT Press), I994.

LLOP-NAYA (20I7) = ARES LlOP-NAYA, La reanàlisi dels minimitzadors negatius en el contínuum romànic pirinenc. Tesis doctoral. Universitat Autònoma de Barcelona, 2017.

Martí (2OIO) = Núria Martí Girbau, The syntax of partitives. Tesis doctoral. Universitat Autònoma de Barcelona, 20 IO.

Martins (2000) = Ana Maria Martins, "Polarity items in Romance: underspecification and lexical change», en S. Pintzuk, G. Tsoulas \& A. Warner (eds.), Diachronic syntax: models and mechanisms, Oxford / New York (Oxford University Press), 2000, págs. I9I-2I9.

Medina Granda (2000) = Rosa Medina Granda, "Expresiones de valor mínimo y polaridad negativa en occitano antiguo. Elementos de comparación con otros romances medievales", Archivum, 50-5I (2000), págs. 79-62.

MeYer-LüBKe (I890-I906) = Wilhem MEYER-LüBKe, Grammaire des langes romanes. III, París (H. Welter), págs. I890-1906.

Milner (i978) = Jean Claude Milner, De la syntaxe à l'interprétation, Paris (Seuil), 1978.

Navarro Tomás (s. D.) = Tomás Navarro tomás (DIr.), Atlas Lingüístico de la Península Ibérica (ALP), Madrid (CSIC), s. d.

Pinto (20I5) = Clara Pinto, "Para a história da negação: o minimizador homem no português antigo", Estudos de Lingüistica Galega, 7 (2015), págs. I09-23, doi:http:// dx.doi.org/IO.I5304/elg.7.2335. 
Postal (2004) = Paul Postal, «The structure of one type of American English vulgar minimizer", en Skeptical linguistic essays, Oxford / New York (Oxford University Press), 2004, págs. I59-172.

RAE-ASALE (2009) = RAE-ASALE, Nueva gramática de la lengua española. I. Madrid (Espasa), 2009.

Roberts \& Roussou (2003) = IAN Roberts \& Anna Roussou, Syntactic change: a minimalist approach to grammaticalization, Oxford / New York (Oxford University Press), 2003.

Rueda (1995) = Mercedes Rueda Rueda, «Los refuerzos de la negación en la literatura medieval española: análisis lingüístico», Contextos, XIII, 25-26 (I995), págs. 93-I33.

San-Segundo-Cachero (aceptado) = Rosabel San-Segundo-Cachero, «Entre la cuantificación y la negación. Los minimizadores y su papel en la negación enfática», Sintagma. Revista de Lingüistica (aceptado).

San-Segundo-Cachero (2017) = Rosabel San-Segundo-Cachero, «Negación enfática y gramaticalización en asturiano: estructuras con ná», Verba. Anuario Galego de Filoloxía, 47 (2017), págs. I-34.

SÁnchez López (1999) = Cristina SÁnchez López, «La negación», en I. BosQue \& V. Demonte (dirs.), Gramática descriptiva de la lengua española. Vol II. Las construcciones sintácticas fundamentales. Relaciones temporales, aspectuales y modales, Madrid (Espasa Calpe), 1999, págs. 256I-2633.

VALLDUVí (1994) = ENRIC VALLduví, «Polarity items, n-words and minimizers in Spanish and Catalan», Probus, 6 (1994), págs. 263-294.

VAN Der Wouden (1994) = TON VAN Der Wouden, «Polarity and 'illogical negation'", en M. Kanazawa \& C. J. Piñon (eds.), Dynamics, polarity and quantification, Stanford, CA (CSLI), 1994, págs. I7-45.

Viejo \& Neira (2008) = Xulio Viejo Fernández \& Ángel Neira Álvarez, «Eslema. Corpus Xeneral de la Llingua Asturiana», 2008, <diog8.edv.uniovi.es/corpus/ adv_search.html>.

Viejo ET Al. (2008) = Xulio Viejo Fernández, Roser Saurí \& Ángel Neira, «Eslema. Towards a corpus for Asturian", en Interoperability between people in the creation of language resources for less-resourced languages. ASALTMIL workshop, Marrakesh (LREC), 2008. <https://scholarspace.manoa.hawaii.edu/bitstream/IoI25/4984/I/4984.pdf>.

Zanuttini (1997) = RafFaella Zanuttini, Negation and clausal structure: a comparative study of Romance languages, New York (Oxford University Press), 1997. 


\section{APÉNDICE. TEXTOS CITADOS (ESLEMA)}

Bodes

Cuentiquinos

Diálogu

Escaecéme

Estatutos

Homes

L'Acampallengua

L'Andecha

L'arna

Mayordomu

Nomes

Pisu

Potestas

Psicokiller

Románticu

Tiempos
Tiempu de bodes, X. M. Sánchez

Cuentiquinos, G. de Lorenzo

Diálogu de Pumarín y otros trabayos de razón local, L. X. Álvarez

Pa nun escaéceme, P. Suárez Coalla

Estatutos y Reglamentu de Réxime Internu del Conseyu de la Mocedá d'Uviéu, P. A. Marín Estrada

Los homes de bronce, X. Fernández

L'Acampallengua hermana a la llingua asturiana y al catalán, X. Santori

L'Andecha d'agora, I. Iglesias

L'arna de San Atanás, P. Rodríguez Medina

Lo que vio'l mayordomu, J. Orton

Los nomes propios

El pisu d'enriba, X. Arbesú Rodríguez

Potestas, X. Arbesú Rodríguez

Psicokiller: obra teatral nun actu, A. C. Díaz

Románticu, M. Rodríguez Cueto

Tiempos de Nublina, X. M. Rodríguez 
